\title{
Calculation of wooden beams reinforced with polymeric composites with modification of the wood compression area
}

\author{
Svetlana Roschina*, Alexey Gribanov, Mikhail Lukin, Mikhail Lisyatnikov and Artem \\ Strekalkin \\ Department of Building Structures, institute of Architecture, Civil Engineering and Power \\ Engineering, Vladimir state university named after Alexander and Nikolay Stoletovs, 87, Gorkogo \\ St.,Vladimir, Russia, 600000
}

\begin{abstract}
Peculiar features of engineering and numerical calculations of wooden beams reinforced with polymeric composites with the local modification of the wood compression area under an insufficient volume of the initial data have been considered in this article. A technique for determining the physical and mechanical properties of materials and a stress-deformation diagram based on experimental investigation has been described. The enlarged algorithm of engineering calculation on the enumerated geometrical characteristics and numerical calculation by the consecutive loading technique and with correcting of the tangent modulus of elasticity at each step instantiated in PC Lira 10.6 has been presented. Analysis of the stress-strain state of wood-composite beams with local modification of wood has been made. Comparative analysis of engineering and numerical calculations has been performed within the limits of the calculated load. Conclusions on the results of theoretical investigations of the proposed beam construction have been formulated and compared with the existing investigations in the field of reinforcement of beam constructions with polymeric composites.
\end{abstract}

\section{Introduction}

Wood is one of the oldest and widely distributed structural material. Combination of high strength, low unit weight, simplicity of processing, a rich forest raw material base of our country allows wood to compete with other traditional materials such as steel and reinforced concrete and even outstrips them by some characteristics such as resistance to chemically aggressive environment, radio transparency, perception of seismic and dynamic loads. The most effective use of wood is found in bending and unbendable structures due to the maximum use of their physical and mechanical characteristics. Oftentimes, to ensure the technological requirements of the designed buildings, to provide fixed construction floor heights and coverings, the development of compact elements with a high load-bearing capacity is required. In addition, during the reconstruction and major repairs of buildings

\footnotetext{
Corresponding author: rsi3@mail.ru
} 
and structures, including cultural heritage sites, some measures are required to ensure the strength and operational reliability of elements with preservation of the architectural appearance. The most effective solution to this issue is to strengthen the elements of the wooden constructions.

The method of reinforcing wooden constructions came into widespread acceptance due to the steel or composite rod reinforcement [1-3]. The drawback of this method is the possibility of its application only for structural reinforcement of the new build construction project. There are methods of strengthening wooden elements with moulded rolled stock [4]. At present, polymeric composites based on glass, coal and aramid fiber have become widely used to reinforce building structures. High physical and mechanical characteristics of the latter combined with the corrosion resistance, low density, radio transparency expand the scope of application of wooden constructions, allow to reduce the labour intensity on the installation of reinforcement systems in large volumes of reconstruction or major repairs, allow to preserve the architectural appearance of structures of architectural monuments. Canvases and fabrics on the polymeric matrix [5-8], lamella [9-13] and laminates [14-16] installed in the tension area of the beam constructions are used as strengthening elements. The effectiveness of such design solutions can be improved by the local modification of the wood compression area by impregnating the polymeric composition provided that the shearing strength of the element in the region of disrupture of the composite material and on the structural supports is ensured $[17,18]$.

From the point of view of structural engineering, the most universal method for estimating the bearing capacity and deformability is the engineering method for calculating wooden constructions on the enumerated geometrical characteristics. This method allows us to determine the stresses and strains within the elastic stage of the material quite adequately. To simulate the work of constructions in the elastic-plastic stage, numerical calculation by the finite element method has been used. The application of numerical methods for solving elastic-plastic problems leads to the emergence of systems of nonlinear algebraic equations. In solving such systems, there may be some difficulties with ramification of solutions and choosing the initial approximation. To solve nonlinear problems, special linearization methods have been developed in which the elastoplastic problem resolves itself into a sequence of elastic.

One of these methods was the method of variable elasticity parameters first proposed by A.A. Ilyushin and further developed in the works of IA. Birger. The method is based on the representation of the stress-strain relationship on the theory of elastoplastic deformations in the form of the generalized Hooke's law in which the elasticity parameters depend on the stress state at a point and are therefore different for different points of the body. [19]

V.V. Petrov's method of successive loading with correcting the tangent modulus at each step for solving nonlinear problems of structural mechanics has come into widespread acceptance $[19,20]$.

These methods have found wide application in solving physically nonlinear problems which is explained by the clear physical meaning of the simulated processes. For example, it is possible to simulate the process of changing the stiffness parameters of the system, caused by the specific factors, on the basis of the method of variable elasticity parameters which fully allows us to describe creep processes in the material of the construction. It is possible to simulate tests of real constructions where the load varies from zero values to values preceding destruction on the basis of the method of successive loading [21].

To calculate and simulate the actual operation of composite wooden constructions, information obtained from the normative documentation (construction rules or technical specifications for the materials used for reinforcement) is often insufficient. Thus, the physical and mechanical characteristics of polymeric composites with fillers from unidirectional tapes, fabrics or cloth to a large extent depend on the bulk weight of the 
bonding matrix. Thus, to simulate the real work of the wood-composite beams, a number of additional experimental investigations may be required to obtain the actual diagrams of the materials strain under the load and their physical and mechanical characteristics.

The purpose of the research is to investigate the stress-strain state of wooden beams reinforced with a unidirectional carbon tape on a tension area span part and the local modification of wood in the compression area on the basis of the results of the engineering calculation and numerical simulation in the "Lira 10.6" software package.

\section{Methodology}

Engineering method of calculation on the enumerated geometrical characteristics and the method of successive loads with the correction of the tangential elastic modulus at each step, implemented in the program complex "Lira 10.6" have been chosen as the methodology. To determine the actual physical and mechanical characteristics of the modified wood and polymeric composite, standard samples have been tested in accordance with GOST 21523.8-93, GOST 16483.10-73 and GOST 11262-2017 respectively.

To assess the effectiveness of modifiers and determine the ultimate compressive strength along the fibers, two series of samples have been prepared according to GOST 16483.10-73 with 5 samples each. A low-viscosity solution based on dimethicryl polyester (DMPE series) has been used as the modifier. The obtained results have been compared with the values for all-wood samples (series D).

Results of the tests of the modified wood samples with the compression along the fibers are presented in Table 1. Statistical processing of the results has been carried out in accordance with GOST 16483.0-89. The deformation diagrams are shown in Figure 1. The increase in strength for the samples of the "DMPE" series has been $46 \%$ compared to the all-wood samples of the "D" series.

Modulus of elasticity of the modified wood on the basis of the compositions presented above has been determined in accordance with GOST 21523.8-93. The method of tensometry using a multichannel measuring complex TDS - 530 load-indicating resistors with a base of $20 \mathrm{~mm}$ has been used as a basis. The general view of the samples during the tests is shown in Figure 1. The test results are shown in Table 2.

Table 1. Test results of the samples under compression along fibers

\begin{tabular}{|c|c|c|c|c|c|c|c|c|c|}
\hline \multirow{2}{*}{$\begin{array}{c}\text { Series and } \\
\text { № }\end{array}$} & \multirow{2}{*}{ Size bxh,mm } & \multirow{2}{*}{$\mathrm{P}, \mathrm{kN}$} & \multirow{2}{*}{$\begin{array}{c}\mathrm{R}, \\
\mathrm{MPa}\end{array}$} & \multicolumn{6}{|c|}{ Static parametres } \\
\hline & & & & $\overline{\mathrm{X}}$ & $S$ & $\overline{\mathrm{S}}$ & $\mathrm{v}, \%$ & $\Delta \mathrm{X}$ & $\bar{P}, \%$ \\
\hline D-1 & $18 \times 19,1$ & 16,90 & 49,15 & \multirow{5}{*}{45,98} & \multirow{5}{*}{2,48} & \multirow{5}{*}{1,11} & \multirow{5}{*}{5,38} & \multirow{5}{*}{3,07} & \multirow{5}{*}{6,68} \\
\hline $\mathrm{D}-2$ & $19,4 \times 19,5$ & 16,90 & 44,67 & & & & & & \\
\hline D-3 & $19,1 \times 18,8$ & 15,50 & 43,17 & & & & & & \\
\hline D-4 & $19,1 \times 20,8$ & 17,87 & 44,99 & & & & & & \\
\hline D-5 & $18,5 \times 19,4$ & 17,21 & 47,94 & & & & & & \\
\hline DMPE-1 & $21,1 \times 19,56$ & 34,00 & 82,54 & \multirow{5}{*}{84,96} & \multirow{5}{*}{4,01} & \multirow{5}{*}{1,79} & \multirow{5}{*}{4,72} & \multirow{5}{*}{4,98} & \multirow{5}{*}{5,86} \\
\hline DMPE -2 & $20,4 \times 20,0$ & 33,43 & 81,69 & & & & & & \\
\hline DMPE -3 & $19,3 \times 20,0$ & 35,10 & 90,80 & & & & & & \\
\hline DMPE -4 & $19,6 \times 21,0$ & 34,01 & 82,27 & & & & & & \\
\hline DMPE -5 & $20,0 \times 19,4$ & 33,95 & 87,51 & & & & & & \\
\hline
\end{tabular}


Table 2. Test results of the samples for the purpose of determining the modulus of elasticity

\begin{tabular}{|c|c|c|c|c|c|c|c|c|}
\hline \multirow{2}{*}{ Series and № } & \multirow{2}{*}{ size bxt,mm } & \multirow{2}{*}{$\mathrm{E}}$, & \multicolumn{6}{|c|}{ Static parametres } \\
\cline { 6 - 9 } & & $\mathrm{GPa}$ & $\overline{\mathrm{X}}$ & $\mathrm{S}$ & $\overline{\mathrm{S}}$ & $\mathrm{v}, \%$ & $\Delta \mathrm{X}$ & $\bar{P}, \%$ \\
\hline DMPEmy-1 & $30,0 \times 29,8$ & 22,05 & & & & & & \\
\hline DMPEmy-2 & $29,6 \times 29,7$ & 21,63 & \multirow{2}{*}{21,56} & 0,52 & 0,30 & 2,43 & 1,30 & 6,03 \\
\hline DMPEmy-3 & $30,1 \times 29,9$ & 21,01 & & & & & & \\
\hline
\end{tabular}

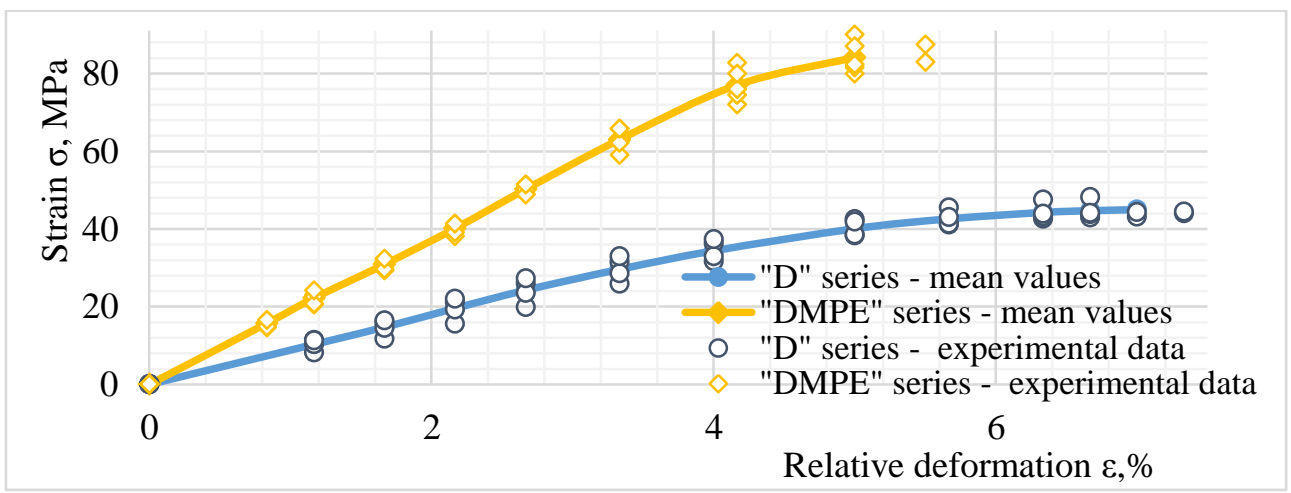

Fig. 1. Diagram of "stain- relative deformations" according to the results of the sample compression testing along fibers according to GOST 16483.10-73.

According to the results of the investigation, the increase of the elasticity modulus of the modified wood "DMPEM" series samples compared to the average values for grade II wood $(\mathrm{E}=10000 \mathrm{MPa})$ has been $53 \%$.

To determine the physical and mechanical characteristics of the polymer composites by the vacuum infusion method, five samples have been prepared on the basis of a unidirectional carbon tape of FibArmTape 430/150 grade and a polymer matrix based on epoxy-diane resin ED-20. The latter has been tested in accordance with GOST 11262-2017 on a REM-100-A-1 tensile testing machine. To prevent the destruction of the samples by the specimen holder, patches of the similar material have been made. The general view of the samples and the nature of the destruction are shown in Figure 2.
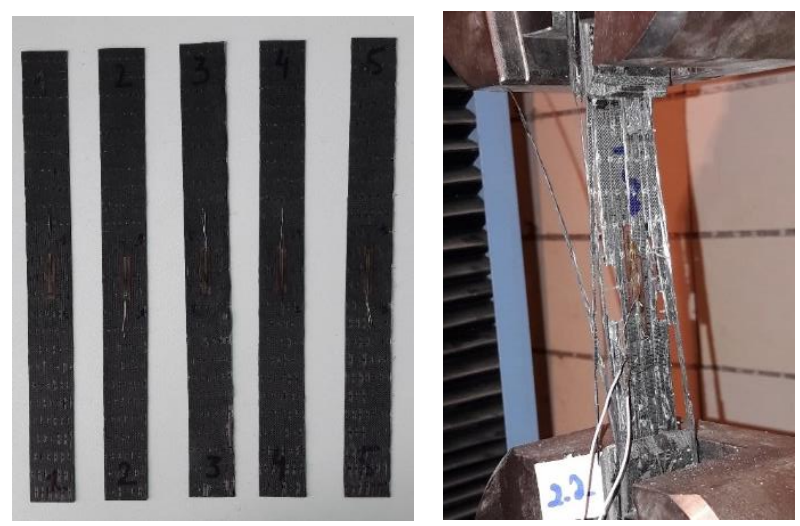

Fig. 2 The general view of the samples from unidirectional carbon tape series No. 2 and the nature of their destruction. 
The limit of the ultimate strength of the samples has been $890 \mathrm{MPa}$, the modulus of elasticity has been $51 \mathrm{GPa}$.

The research of the stress-strain state of the wood-composite beam structures, shown in Figure 3 has been conducted within the framework of the investigation based on the obtained data. All-wood beams with the cross section of 100x200 (h) $\mathrm{mm}$ (grade DB) have been used as the model and blank. Reinforcement of the construction has been accomplished by sticking the unidirectional carbon tape of the brand FibArm Tape 430/150 in the element stretched area on the span parts onto an epoxy matrix based on ED-20 resin with the reinforcing material being placed on the lateral edges of the cross section to reduce the effect of the most dangerous wood defects in the form of edge knots, cut fibers of the twisted growth and others (the DKB brand). The third method of reinforcement includes, in addition to the second one, a local modification of the wood of the compression area carried out at a height of $30 \mathrm{~mm}$ by impregnating the composition based on dimethacrylic polyester (DKMB grade).

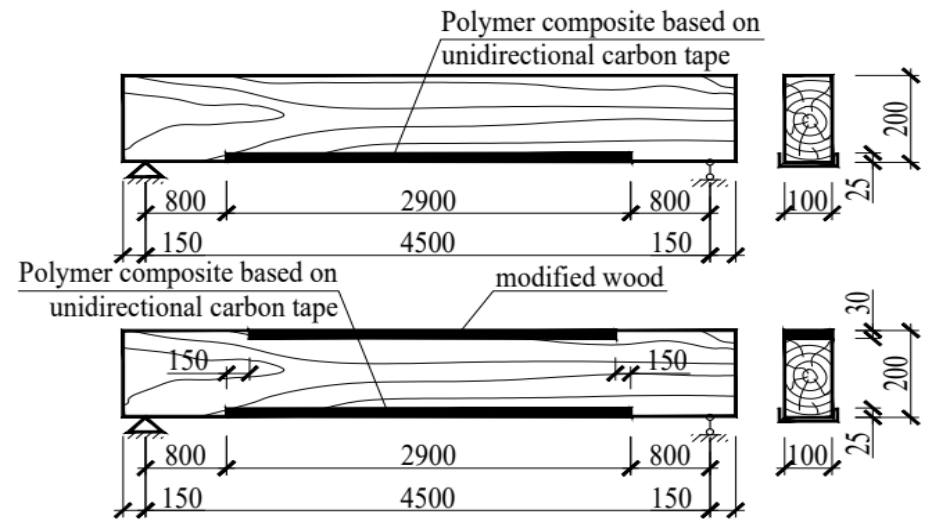

Fig. 3. Wood-composite beam structures

Calculation of the wood-composite structures according to the enumerated geometrical characteristics has been performed in several stages. The first stage includes laying down the enumerated geometric characteristics of the composite sections. To do this, the reduction coefficient $\left(\mathrm{n}_{1}\right)$ and the enumerated values of the inertia moment $\left(\mathrm{I}_{\text {пр }}\right)$ and the moment of resistance $\left(\mathrm{W}_{\text {пр }}{ }^{\mathrm{c}}\right)$ according to formulas 1-3 is determined. Then a check for normal and shear stresses is run.

$E_{D}$ - modulus of elasticity of wood;

$$
\begin{gathered}
n_{1}=\frac{E_{\mathrm{a}}}{E_{D}} \\
I_{P}=I_{D}+n_{1} \cdot \sum_{i=1}^{m} I_{k i} \\
W_{P}^{\mathrm{c}}=\frac{I_{P}}{h_{c}}
\end{gathered}
$$

$\mathrm{E}_{\mathrm{a}}$ - modulus of elasticity of the reinforcing component;

$I_{D}$ - moment of inertia of the wooden section;

$\mathrm{h}_{\mathrm{c}}$ - height of the compressed zone of the cross section;

Numerical calculation of the wood-composite beams has been carried out in the program complex "Lira 10.6" in a physically linear and nonlinear formulation. To model 
the actual work of construction in the elastic-plastic stage, piecewise linear functions of the used materials have been employed. For the conventional wood, the enumerated BelyankinPragerw diagram has been used, which characterizes the work of the pure wood behaviour along fibers in tension and compression. The actual diagrams of deformation of the modified wood under compression along fibers and tensioning the polymeric composites have been obtained on the basis of the results of the above experimental investigations. The conventional and modified wood is modeled by physically nonlinear and universal spatial 8-node isoparametric finite elements, the polymer composite by spatial eight-node isoparametric finite elements with the ultimate strength of $890 \mathrm{MPa}$. The results of the numerical calculations are shown in Fig. 4. The loading of the beam structure has been carried out by a uniformly distributed load throughout the span. The design load on the wood-composite beam has been determined according to the strength condition of the normal cross-sections in the middle of the span and amounted $-5.13 \mathrm{kN} / \mathrm{m}$ for the DB beam, $5.57 \mathrm{kN} / \mathrm{m}$ for the DKB beam and $7.37 \mathrm{kN} / \mathrm{m}$ for the DKMB beam.

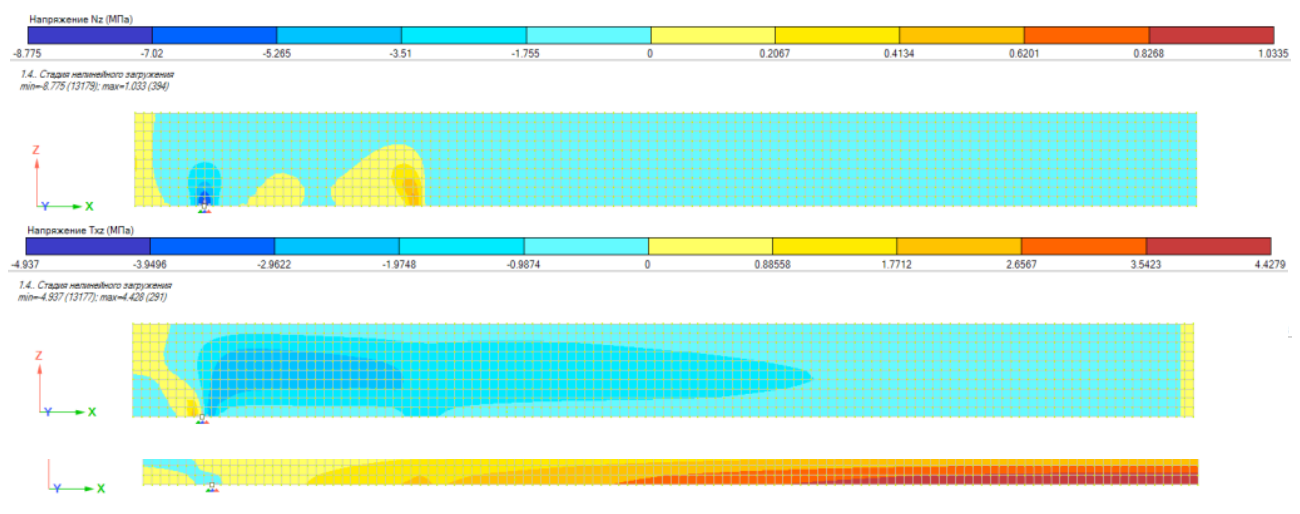

Fig. 4. Isopoles on the halfspan of the DKMB series beam: a) normal stresses $\sigma x, M P a ; b)$ normal stresses $\sigma \mathrm{z}, \mathrm{MPa} ; \mathrm{c})$ shear stresses $\tau \mathrm{xy}, \mathrm{MPa}$

Destruction of the DKB wood-composite beam took place at normal cross-sections in the middle of the span when the ordinary wood reached the ultimate compressive strength along the fibers under the linear load of $28.4 \mathrm{kN} / \mathrm{m}$ on the beam. Beams DKMB - 34,1 $\mathrm{\kappa H} /$ $\mathrm{m}$. Results of the investigation have shown that a complex stress state including the simultaneous action of the normal stress along and across the fibers and tangential stresses with the resultant at an angle to the fibers occurs in the zone of breakage of the composite material. The value of the working stresses for low beams in the considered cross section with the ratio of $\mathrm{h} / \mathrm{b}<4,1 / \mathrm{h}=1 / 20 \ldots 1 / 30$ does not exceed the maximum allowable and the component damage occurs according to normal stresses in the middle of the span. In the limit state a significant redistribution of forces occurs between the reinforcing material, modified and conventional wood, while the polymeric composite reduces the stress level in the wood of the tension area from six fold to eightfold. Destruction of the all-wood beam takes place with a running ultimate breaking load, equal to $9,18 \mathrm{kN} / \mathrm{m}$ in the normal crosssections in the middle of the span. The actual ultimate breaking load for all-wood beams can have significant differences from the results obtained in the numerical simulation, due to the presence of defects in the tension area.

Based on the results of engineering and numerical calculations, the "load-stress" and "load-strain" relationships are presented within the limits of the design loads respectively in Figures 5 and 6. 
a)

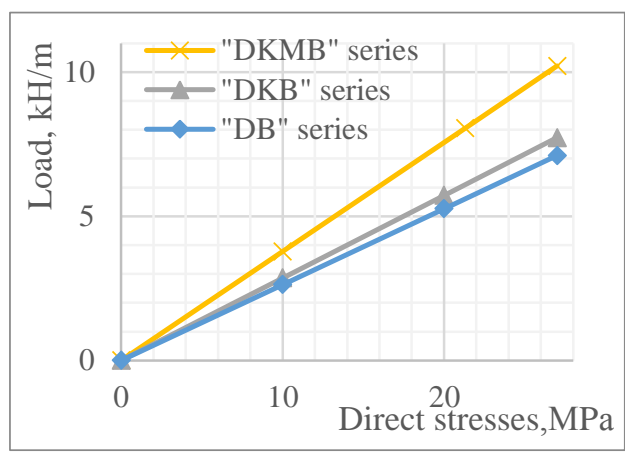

b)

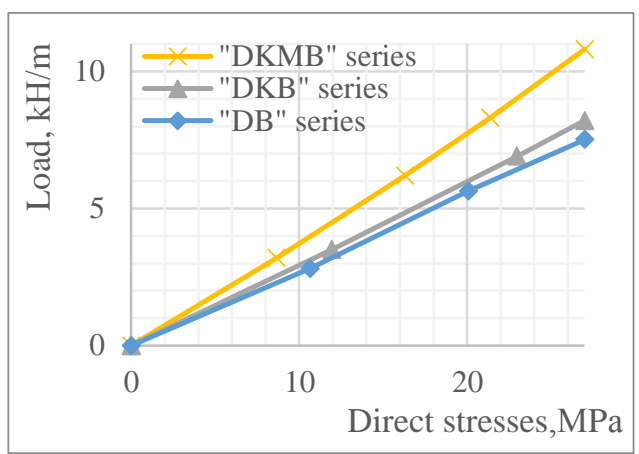

Fig. 5. Load-direct stresses for wood-composite beams based on the results of: a) engineering calculation; b) numerical calculation

a)

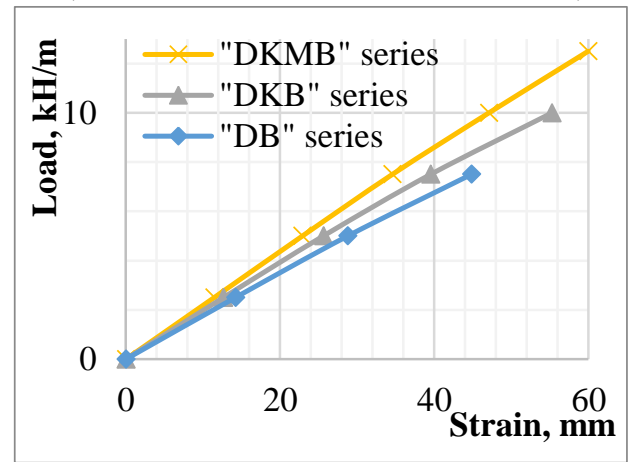

b)

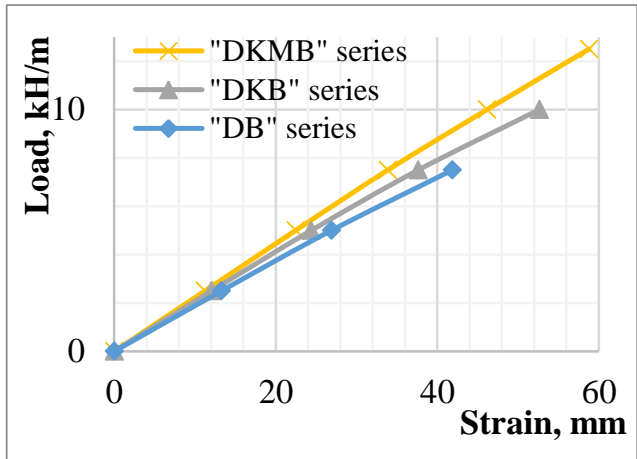

Fig. 6. Dependences "load-strain" for wood-composite beams based on the results of: a) engineering calculation; b) numerical calculation

\section{The following conclusions based on the results of the studies can be made:}

1. The proposed numerical model with due account for the actual physicomechanical characteristics of materials makes it possible to model the real work of wood-composite beams at all stages of the stress-strain state with a sufficient accuracy.

2. High convergence of the results of engineering and numerical calculations has been established. The discrepancy between the results of the strength has been $3-6 \%$, of the deformation - 4-9\%.

3. An increase in the strength of the wood-composite beam DKB by $15 \%$, DKMB - by $33 \%$ has been established. The increase in the stiffness (decrease in deformability) by $6 \%$ and $18 \%$ respectively.

4. Local modification of the wood compression area significantly increases the efficiency of the single reinforcement of the tension area.

The deformation curve of the beam structures under consideration and the obtained fracture pattern are strongly consistent with the results of the investigations devoted to the single reinforcement of low wooden beams by unidirectional carbon tapes on the polymer matrix. The advantage of the proposed reinforcement option is to increase the efficiency of single reinforcement of wooden beams due to the local modification of wood of the 
compression area. In addition, the local modification of wood allows to improve the operational factors of beam constructions by increasing biostability, reducing water absorption and preserving the architectural appearance.

\section{References}

1. S. Roschina. Wood journal. 2. 12. (2008)

2. M Gary, C. Whelan. Constr. and build. mat. 52. 209-220 (2014)

3. M. Fossetti, G. Minaft, M. Papia. Constr. and build. mat. 95. 54-64 (2015)

4. S. Roschina, E. Smirnov, M. Sergeev, M. Lukin, P. Shokhin. Patent for utility model RUS 118333

5. Y.Nadir, P. Nagarajan, M. Ameen, M. Arif. Constr. and build. mat. 112. 547-555 (2016)

6. L. Basterra, J. Balmori, L. Morillas, L. Acuña, M. Casado. Constr. and build. mat. 154. 914-920 (2017)

7. S. Roschina, M. Sergeev, A. Lukina, M. Lisyatnikov. Scientific and Technical Herald of the Volga Region. 2. 189-192. (2012)

8. S. Roschina, M. Sergeev, M. Lukin, P. Shokhin. Forest journal . 3. 103-107. (2013)

9. A. D’Ambrisi, F. Focacci, R. Luciano. Comp. struct. 108. 720-728 (2014).

10. M. Khelifa, A. Celzard Comp. struct. 111. 393-400 (2014).

11. Y. Li., Y. Xie, M. Tsai. Constr. and build. mat. 23. 411-422 (2012).

12. A. Jesus, J. Pinto, J. Morais. Constr. and build. mat. 35. 817-828 (2012).

13. A. Andre, R. Kliger, R. Olsson. Constr. and build. mat. 41. 790-800 (2013).

14. M. Morales-Conde, C. Rodrhguez-Licon, P. Rubio-de Hita. Constr. and build. mat. 96. 461-472 (2015).

15. K. Andor, A. Lengyel, R. Polgár, T. Fodor, Z. Karácsonyi Constr. and build. mat. 99. 200-207 (2015).

16. A. Zhou, L. Tam, Z. Yu, D. Lau. Composits. Part B 71. 63-73 (2015)

17. S. Roschina, M. Lukin A. Lukina, M. Lisyatnikov. Scientific and Technical Herald of the Volga Region. 4. 182-184. (2014)

18. S. Roschina, M. Lukin A. Lukina, M. Lisyatnikov. Logging journal. 3. 183-190. (2015)

19. P. Iliin Numerical Methods for Solving the Problems of Building Mechanics: A Reference Manual (Hight school, Moscow, 1990)

20. V. Presekin. Fundamentals of the finite element method in mechanics of deformable bodies (NgTU, Novosibirsk, 2010)

21. S. Gorodetsky The finite element method in the design of transport structures (Transport, Moscow, 2000) 